\title{
Uso clínico do strain rate na ecocardiografia sob estresse em paciente assintomática com fatores de risco para doença cardiovascular: relato de caso
} Strain rate clinical use associated with stress echocardiography in asymptomatic patient with cardiovascular risk factors: case report

O ecodopplercardiograma transtorácico é um exame de imagem de ampla aplicabilidade clínica que, apesar do requerimento de grande experiência do operador e de possibilidade de janelas acústicas ruins, permite a obtenção de muitas informações de maneira não invasiva, sem riscos de ionização para o paciente, com baixo custo. Quando realizado sob estresse físico ou farmacológico, torna-se uma opção com apropriada acurácia diagnóstica para investigação de isquemia miocárdica. Dentre os recursos disponíveis, o strain rate tem se mostrado útil na detecção de alterações incipientes da função sistólica segmentar, inclusive em pacientes portadores de hipertensão arterial sistêmica e doença arterial coronária. O presente trabalho demonstra o caso de uma paciente do gênero feminino assintomática que, após a submissão ao ecodopplercardiograma sob estresse farmacológico, apresentou hipertrofia do ventrículo esquerdo e strain rate alterado, mesmo sem alterações segmentares de motilidade miocárdica.

Palavras-chave: ecocardiografia doppler; ecocardiografia sob estresse; doença de artéria coronariana; hipertensão; isquemia miocárdica; fatores de risco.

\begin{abstract}
The transthoracic Doppler echocardiography is an image exam of broad clinical applicability that, despite the requirement of great experience of the operator and the possibility of poor acoustic windows, allows the collection of a lot of information non-invasively, without ionization risks to the patient, with low cost. When performed under physical or pharmacological stress, it is an option with appropriate diagnostic accuracy for myocardial ischemia screening. Among the available resources, strain rate has proven to be useful for detecting incipient changes in segmental systolic function, including patients with systemic arterial hypertension and coronary artery disease. This work demonstrates the case of an asymptomatic female patient that, after submission to Doppler echocardiography under pharmacological stress, showed left ventricular hypertrophy and strain rate changes, even without segmental changes of myocardial motility.
\end{abstract}

Keywords: echocardiography, Doppler; echocardiography, stress; coronary artery disease; hypertension; myocardial ischemia; risk factors.

\section{INTRODUÇÃO}

O ecodopplercardiograma transtorácico é um exame complementar com ampla aplicabilidade clínica. Requer experiência do operador e pode ter como possível limitação condições torácicas desfavoráveis com janelas acústicas inadequadas, mas trata-se de exame de baixo custo, com muitas informações obtidas de modo não invasivo, sem riscos de ionização para o paciente. Utiliza os princípios da ultrassonografia, através do modo $\mathrm{M}$, bi e tridimensional, para a avaliação dinâmica de aspectos anatômicos e funcionais do coração e grandes vasos. Com o recurso do Doppler, permite avaliação de aspectos hemodinâmicos dessas estruturas. ${ }^{1}$

Em portadores de hipertensão arterial sistêmica (HAS), a avaliação ecodopplercardiográfica é recomendada para pacientes que apresentem suspeita de hipertrofia ventricular esquerda (HVE), três ou mais fatores de risco para doença cardiovascular e suspeita de disfunção ventricular, além da reavaliação daqueles pacientes sem alteração do quadro clí-

'Pontifícia Universidade Católica de São Paulo, Faculdade de Ciências Médicas e da Saúde - Sorocaba (SP), Brasil.

Contato: ivanff@live.com

Recebido em 01/11/2016 - Aceito para publicação em 20/02/2017. 
nico. ${ }^{2}$ Pacientes hipertensos com HVE podem cursar com aumento da massa miocárdica, com padrão de remodelamento concêntrico inicial. ${ }^{3}$ A presença de disfunção diastólica pode representar lesão de órgão alvo incipiente, por conta de alterações na micro ou na macrocirculação coronariana e possível fibrose intersticial. ${ }^{4}$

Em portadores de doença arterial coronariana (DAC), esse método, quando realizado sob estresse físico ou farmacológico, torna-se opção com apropriada acurácia diagnóstica (equipara-se à cintilografia de perfusão miocárdica) em indivíduos sintomáticos com teste ergométrico não diagnóstico e em indivíduos com quadro clínico não sugestivo de isquemia miocárdica, mas com teste ergométrico positivo ou duvidoso. ${ }^{5} \mathrm{O}$ desequilíbrio entre a oferta e a demanda de oxigênio no miocárdio pode induzir alterações da contratilidade segmentar e espessamento endocárdico, correspondendo, assim, à presença de isquemia miocárdica. ${ }^{6}$

$\mathrm{Na}$ ecodopplercardiografia com estresse farmacológico, destaca-se a possibilidade da utilização de adenosina, dipiridamol e dobutamina, sendo a última a mais amplamente difundida. Quanto à dobutamina, utiliza-se a infusão contínua, na dose inicial de $5 \mathrm{mcg} / \mathrm{kg} / \mathrm{min}$, com incrementos para 10, 20, 30 e $40 \mathrm{mcg} / \mathrm{kg} / \mathrm{min}$, a cada 3 minutos. Associa-se atropina e/ou esforço isométrico, conforme a necessidade de cada caso, com o objetivo de alcançar a frequência cardíaca (FC) programada previamente, que geralmente corresponde a $85 \%$ da FC máxima prevista (220-idade). Durante o período do exame, o ritmo e a FC do indivíduo são monitorizados por derivação eletrocardiográfica contínua. A pressão arterial e um eletrocardiograma (ECG) de 12 derivações são registrados. A cada etapa do protocolo, a contratilidade miocárdica é analisada e registros de todos os segmentos miocárdicos são realizados. ${ }^{7}$

Atualmente, dispõe-se do recurso chamado strain rate (SR), cuja definição consiste em um índice de deformação miocárdica regional e global, mensurados através da técnica de rastreamento de pontos (speckle-tracking). Mostra-se útil na detecção de alterações incipientes da função ventricular segmentar. A técnica permite a quantificação de deformação miocárdica em planos longitudinal, circunferencial e radial ao medir a velocidade tecidual e torção ventricular sem a dependência do ângulo de incidência do ultrassom, identificando pontos intramiocárdicos brilhantes (speckles) e os acompanhando durante o ciclo cardíaco. ${ }^{8}$

Em hipertensos com HVE, o SR em plano longitudinal pode estar reduzido em comparação com indivíduos controle, pela alteração na estrutura do ventrículo esquerdo (VE). $\mathrm{Na}$ DAC, o SR longitudinal pode alterar-se primeiramente em decorrência das anormalidades de perfusão na região subendocárdica, a qual sofre maior deformidade durante a sístole em relação à região subepicárdica, implicando em aumento na pressão e no consumo de oxigênio. ${ }^{8}$

\section{RELATO DE CASO}

Paciente do sexo feminino, branca, com 71 anos, hipertensa, obesa grau I (índice de massa corporal - IMC $=34,5$ ) e hipotireoidea assintomática. Foi admitida no serviço de ecodopplercardiografia do hospital da Unimed Sorocaba, São Paulo, com o intuito de ser submetida ao exame de ecodopplercardiograma sob estresse para investigação de isquemia miocárdica, conforme indicação de seu médico assistente. Faz uso regular de levotiroxina $100 \mathrm{mcg} / \mathrm{dia}$, losartana $100 \mathrm{mg} / \mathrm{dia}$, hidroclorotiazida $25 \mathrm{mg} / \mathrm{dia}$ e vitamina D.

Após jejum de 4 a 6 horas, esclarecimento de todas as dúvidas referentes à rotina do exame e assinatura de termo de consentimento livre e esclarecido (TCLE), a paciente foi submetida à infusão contínua de dobutamina em doses crescentes a cada três minutos, atropina intravenosa e esforço isométrico (Figura 1).

A FC e o ECG foram monitorados continuamente, com registro da pressão arterial (PA) e ECG de 12 derivações ao final de cada etapa do protocolo. A FC submáxima calculada foi de 127 batimentos por minuto.

No início do exame foram mensurados os diâmetros diastólico e sistólico finais do VE, a espessura do septo interventricular e da parede posterior do VE na diástole, o diâmetro do átrio esquerdo (AE) e o diâmetro da raiz da aorta. Os valores encontrados, em milímetros, foram, respectivamente, 48, 29, 12, 11, 42 e 34. A fração de ejeção foi estimada pelo método de Teicholz em $68 \%$. Esses dados permitiram concluir que o átrio esquerdo (AE) apresenta discreto aumento, o VE demonstra hipertrofia concêntrica discreta, a função sistólica global do VE está preservada, sem alteração contrátil segmentar em repouso, com disfunção diastólica grau I (leve) do VE. A análise do aparelho valvar não encontrou alterações significativas. O ECG inicial demonstrava ritmo sinusal com discreta alteração inespecífica da repolarização ventricular, difusa. A PA inicial foi de 156x87 mmHg e a FC de 52 batimentos por minuto.

Após $30 \mathrm{mcg} / \mathrm{kg} / \mathrm{min}$ de dobutamina, 0,5 mg de atropina e exercício isométrico, atingiu-se o pico do estresse, com hipercinesia de todos os segmentos do VE, sem alterações patológicas adicionais do segmento ST e da onda $\mathrm{T}$ ao ECG. A PA foi de 117x60 mmHg e a FC de 129 batimentos por minuto.

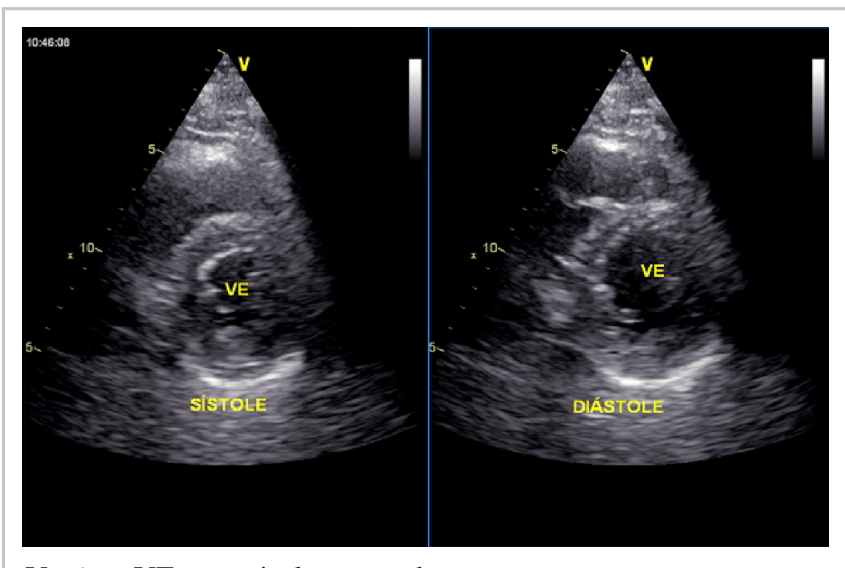

$\mathrm{V}$ : view; VE: ventrículo esquerdo.

Figura 1. Ecocardiografia basal do ventrículo esquerdo. 
Apesar do aspecto de espessamento endocárdico normal em todos os segmentos do VE ao modo bidimensional, após análise segmentar com o recurso do SR longitudinal de pico sistólico (na fase de recuperação imediata), foi encontrada média global de $-19,6 \%$ (normal), mas com índices alterados em região médio-basal da parede lateral do VE (Figuras 2 e 3).

A fase de recuperação cursou com PA de $124 \times 77$ mmHg e FC de 50 batimentos por minuto.

Considerando-se os dados clínicos (ausência de dor torácica ou outros sintomas de natureza cardiológica), ausência de alterações isquêmicas ao eletrocardiograma, espessamento

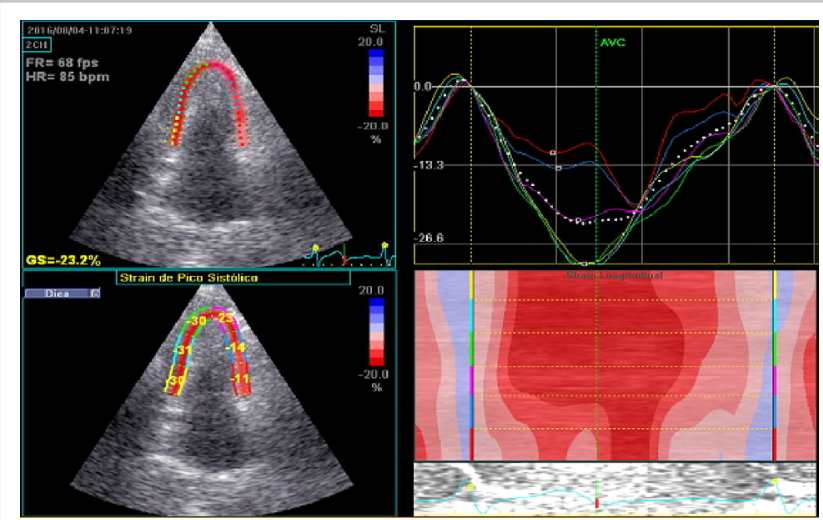

RF: frame rate; HR: heart rate; GS: strain longitudinal global; AVC: aortic valve closure; $\mathrm{CH}$ : chamber; SL: strain longitudinal.

Figura 2. Determinação do strain rate do ventrículo esquerdo.

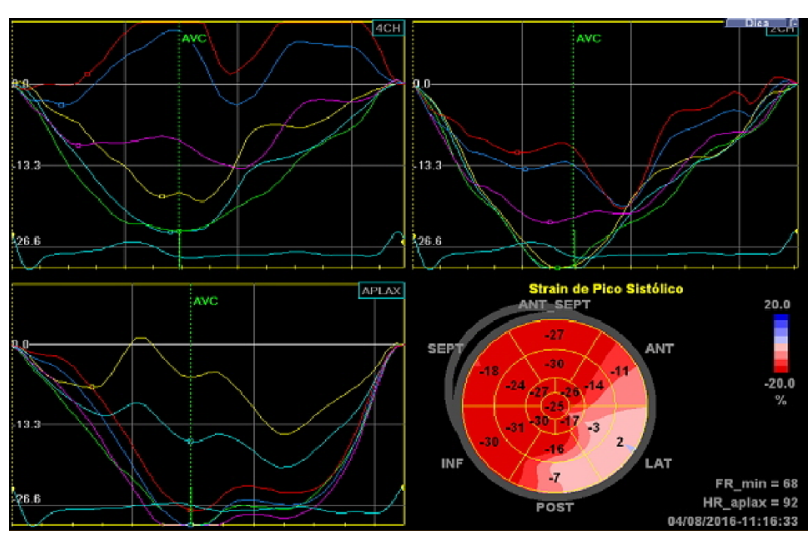

AVC: aortic valve closure; $\mathrm{CH}$ : chamber; APLAX: corte ecocardiográfico apical em cinco câmaras; ANT_SEPT: anterior septal; SEPT: septal; ANT: anterior; LAT: lateral; INF: inferior; POST: posterior; FR_min: frame rate mínimo; HR_aplax: heart rate no momento da imagem bidimensional. AVC: aortic valve closure; $\mathrm{CH}$ : chamber; APLAX: corte ecocardiográfico apical em cinco câmaras; ANT_SEPT: anterior septal; SEPT: septal; ANT: anterior; LAT: lateral; INF: inferior; POST: posterior; FR_min: frame rate mínimo; HR_aplax: heart rate no momento da imagem bidimensional.

Figura 3. Strain rate alterado na região médio-basal da parede lateral do ventrículo esquerdo. endocárdico normal em todos os segmentos do VE e média global normal do SR longitudinal de pico sistólico, concluise que não há evidências de isquemia miocárdica. Porém, foram encontrados índices alterados do SR longitudinal do pico sistólico, em segmentos médio-basal da parede lateral do $\mathrm{VE}$, que podem representar presença de fibrose relacionada à miocardiopatia hipertensiva e não necessariamente isquemia miocárdica relacionada à DAC.

\section{DISCUSSÃO E CONCLUSÃO}

A DAC relaciona-se à disfunção endotelial, que pode ser secundária aos fatores de risco para doença cardiovascular (DCV), que se dividem em não modificáveis (idade, sexo masculino, baixa condição socioeconômica e história de DCV precoce) e modificáveis (HAS, diabetes mellitus, sobrepeso/obesidade, dislipidemias, tabagismo, sedentarismo, ingestão de álcool e fatores psicossociais). A HAS destaca-se nesse cenário pela sua elevada prevalência na população mundial. ${ }^{9}$ A idade superior a 55 anos duplica o risco de DAC. ${ }^{10}$ Logo, a paciente apresenta condições para desenvolver doenças cardiovasculares, e por isso a indicação da pesquisa pelo assistente.

O protocolo mais utilizado para realização de ecodopplercardiografia sob estresse com dobutamina consiste na infusão inicial de $5 \mathrm{mcg} / \mathrm{kg} / \mathrm{min}$ com incrementos para 10 , 20,30 e $40 \mathrm{mcg} / \mathrm{kg} / \mathrm{min}$ em intervalos de 3 minutos até atingir a FC submáxima prevista. Pode-se associar atropina intravenosa, em dose de $0,25 \mathrm{mg} / \mathrm{min}$ até $1 \mathrm{mg}$, geralmente para melhora da sensibilidade em pacientes que fazem uso de betabloqueadores ou que são portadores de lesão uniarterial, além de melhorar o esforço isométrico. Ao término do protocolo do estresse, pode-se utilizar betabloqueador, como esmolol intravenoso, para retorno aos padrões pré-estresse e controle de possível isquemia que tenha sido induzida. ${ }^{7} \mathrm{O}$ protocolo utilizado no exame do caso em questão está de acordo com o preconizado na literatura especializada.

Dentre os achados da ecodopplercardiografia basal, a HVE concêntrica, o aumento do volume atrial esquerdo e a disfunção diastólica grau I podem ser decorrentes da HAS. ${ }^{11}$

As alterações de repolarização ventricular ao ECG são inespecíficas e não necessariamente relacionam-se à isquemia miocárdica ou à HVE. ${ }^{12}$

A aplicabilidade clínica do SR é consistente em algumas doenças cardiovasculares como HAS, disfunção sistólica, disfunção diastólica, doenças isquêmicas do miocárdio, valvopatias e cardiomiopatias.

Na ecodopplercardiografia por estresse, esse recurso permite redução da subjetividade na avaliação, com incremento da acurácia diagnóstica e melhora na sensibilidade para pesquisa de doenças isquêmicas do coração. Em hipertensos portadores de HVE e fibrose, observa-se alterações nos índices do $\mathrm{SR}$, mesmo na ausência de isquemia miocárdica. Em pacientes com insuficiência cardíaca, o SR demonstrou alta concordância com a presença de fibrose miocárdica. ${ }^{13}$ Logo, a paciente em questão pode apresentar áreas difusas de fibrose, mais notadamente em região médio-basal da parede lateral do VE, mesmo sem alterações segmentares da contratilidade miocárdica. 


\section{REFERÊNCIAS}

1. Chen MY, Pope TL, Ott DJ. Basic radiology. $2^{\mathrm{a}}$ ed. New York: Lange Medical Books/McGraw-Hill; 2011.

2. Camarozano A, Rabischoffsky A, Maciel BC, Brindeiro Filho D, Horowitz ES, Pena JL, et al. Diretrizes das indicações da ecocardiografia. Arq Bras Cardiol. 2009;93(6 Suppl 3):e265-302.

3. Otto CM. Fundamentos de ecocardiografia clínica. $5^{\mathrm{a}}$ ed. Rio de Janeiro: Elsevier Health Sciences Brazil; 2015.

4. Matos-Souza JR, Franchini KG, Nadruz Junior W. Hipertrofia ventricular esquerda: o caminho para a insuficiência cardíaca. Rev Bras Hipertens. 2008;15(2):71-4.

5. Cesar LA, Ferreira JF, Armaganijan D, Gowdak LH, Mansur AP, Bodanese LC. Diretriz de doença coronária estável. Arq Bras Cardiol. 2014;103(2 Suppl 2):1-59.

6. Oliveira JL, Barreto MA, Silva AB, Sousa AC. Ecocardiografia sob estresse em coronariopatia. Rev Bras Cir Cardiovasc. 2004;19(1):55-63. DOI: 10.1590/ S0102-76382004000100011

7. Sanchez-Osella OF, Arraes WA, Cardoso RL, Melo MB, Carranza GP, Silva TB. Ecocardiografia sob estresse farmacológico com dobutamina em baixa dose associada a exercício isométrico e atropina precoce: relato da experiência com novo protocolo. Rev Bras Ecocardiogr Imagem Cardiovasc. 2013;26(2):98-104.
8. Abduch MC, Alencar AM, Mathias Jr. W, Vieira ML. Cardiac mechanics evaluated by speckle tracking echocardiography. Arq Bras Cardiol. 2014; 102(4):40312. DOI: $10.5935 / \mathrm{abc} .20140041$

9. Simão AF, Precoma DB, Andrade JP, Correa Filho H, Saraiva JFK, Oliveira GMM, et al. Diretriz Brasileira de prevenção cardiovascular. Arq Bras Cardiol. 2013;101(6 Suppl 2):1-63. DOI: 10.5935/abc.2013S012

10. Gama GG, Mussi FC, Guimarães AC. Revisando os fatores de risco cardiovascular. Rev Enferm UERJ. 2010;18(4):650-5.

11. El Aouar LM, Meyerfreud D, Magalhães P, Rodrigues SL, Baldo MP, Brasil Y, et al. Relationship between left atrial volume and diastolic dysfunction in 500 Brazilian patients. Arq Bras Cardiol. 2013;101(1):52-8. DOI: 10.5935/abc.20130109

12. Sociedade Brasileira de Cardiologia. Diretrizes da Sociedade Brasileira de Cardiologia sobre Análise e Emissão de Laudos Eletrocardiográficos. Arq Bras Cardiol. 2009;93(3 Suppl 2):1-19.

13. Almeida AL, Gjesdal O, Mewton N, Eui-Young C, Teixido-Tura G, Yoneyama K, et al. Speckle-Tracking pela ecocardiografia bidimensional: aplicações clínicas. Rev Bras Ecocardiogr Imagem Cardiovasc. 2013;26(1):38-49. 